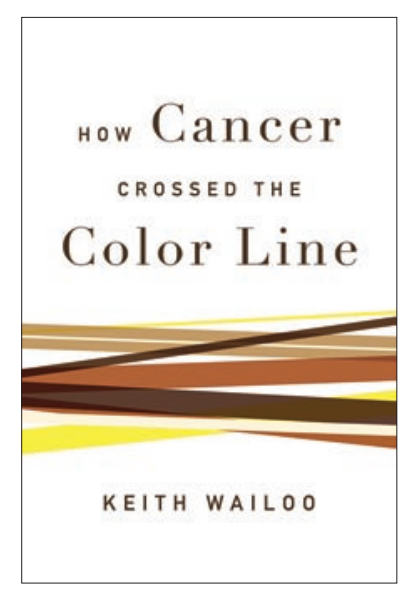

\title{
How cancer crossed the color line
}

\author{
Keith Wailoo \\ Oxford University Press. New York, New York, USA. 2011. \\ 264 pp. \$27.95. ISBN: 978-0-195-17017-7 (hardcover).
}

Reviewed by Katrina Armstrong

University of Pennsylvania, Philadelphia, Pennsylvania, USA.

E-mail:karmstro@mail.med.upenn.edu
The he unequal burden of cancer among minority populations has been so prominent in public discourse about cancer in the US over the last several decades that it is often hard to remember that the current dialogue represents a substantial shift from the dialogues about race and cancer in the past. The book, How Cancer Crossed the Color Line, describes the path by which the current dialogue has grown out of the changing and often problematic understanding of the relationship between race and cancer in the US over the last 90 years. Written by Keith Wailoo, the director of the Center for Race and Ethnicity at Rutgers University, this well-researched book demonstrates the importance of framing our current approach to cancer control in a historical context and should be of interest to cancer researchers as well as those seeking a broader understanding of how social forces can shape scientific discourse.

This book is largely devoted to telling the story of the relationship between cancer and race in the US since the early 1900s. In the 1920s, cancer was seen as an affliction of white women, and the book follows the growing epidemiological interest in studying variation in incidence and outcomes across groups as well as the politicization of both race and cancer in the 1960s and 1970s. It ends with case studies of PSA screening and the Marin county breast cancer cluster in the 1990s. Primarily, the story focuses on the changing picture of the relationship between African-Americans and cancer, though other group-based observations are also summarized.

Within this historical perspective, the narrative highlights several themes that have continued relevance to the latest battles in our "War against Cancer." In the early part of the 20th century, women's reproductive cancers occupied nearly all of the social awareness about the disease, leading to hypotheses about the relationship between cancer incidence and women's reproductive roles. An explanatory model that linked cancer incidence to the changing social roles of women was reinforced by the observation that African-American women experienced lower rates of cancer, and it was speculated that this was related to the fact that women remained closer to their "natural" roles in those communities. Toward the middle of the century, differences in cancer incidence between racial and ethnic groups became of increasing interest, as some of the first epidemiologic studies of cancer rates provided surprising and vivid accounts of the population variation. These differences spawned new hypotheses about the causes of cancer, including the role of diet and environmental exposure. These studies also brought more attention to the debate about the role of inadequate access to care and poor reporting infrastructure in masking the true burden of cancer among minorities.

By the last half of the century, cancer awareness had grown substantially as had the challenges in finding effective prevention and treatment. Public desperation for effective cancer treatment and skepticism about the intellectual establishment led to the increasing politicization of cancer at the same time that race became even more politicized in the US. Following the report by Howard University researchers in 1972, demonstrating the dramatic increase in the cancer burden among African Americans, the social and political activity around race and cancer coalesced in a growing focus on racial disparities in cancer incidence.
The story of race and cancer in the US is important, and there is a sense of achievement in the publication of this book. It offers an overdue perspective both on the history of cancer in our society and on how we come to define and understand differences across racial groups in any disease. However, Wailoo writes as a historian for a social science audience, and the book's impact in those fields is likely to be far greater than that in the biomedical world. Several topics receive too little attention to be satisfying for most cancer researchers, particularly those interested in disparities. The debate about the role of biology/genetics is glossed over with little insight into the revolutions in molecular genetics that continue to inform and influence the discourse surrounding race and cancer. Similarly, the role of health care quality, encompassing prevention, early detection, and treatment, receives remarkably little attention given its prominence in the lay and medical press about cancer disparities. Wailoo identifies one of the fundamental paradoxes of racial disparities research (i.e., that racial identity must be simplified and categorized if disparities are to be measured and monitored) but has very little to offer as a way forward in this area.

Science grows from attempts to explain observations. The history of race and cancer in the US shows how the interpretations of some observations can be been influenced by the sociopolitical environment of any period and can, in turn, influence the sociopolitical environment in the future. In the end, the main reason to read How Cancer Crossed the Color Line has less to do with gaining a comprehensive understanding of race and cancer in the US and more to do with catching yet another glimpse of the fundamental truth that science and society are inextricably linked. 